Recibido: 14/01/2021 --- Aceptado: 15/04/2021 --- Publicado: 12/04/2021

\title{
FANDOMTELEVISIVO. ESTUDIO DE SU IMPACTO EN LA ESTRATEGIA DE COMUNICACIÓN EN REDES SOCIALES DE NETFLIX
}

\section{TELEVISION FANDOM. STUDY OF ITS IMPACT ON THE NETFLIX'S SOCIAL MEDIA COMMUNICATION STRATEGY}

(73) Alba-María Martínez-Sala': Universidad de Alicante. España. albamaria.martinez@ua.es España.

Almudena Barrientos-Báez: Escuela Universitaria de Turismo Iriarte. almudenabarrientos@iriarteuniversidad.es

David Caldevilla-Domínguez: Universidad Complutense de Madrid. España. davidcaldevilla@ccinf.ucm.es

Agradecimientos. El presente trabajo contó con la financiación de los grupos de investigación Marketing Experiencial, Eventos y Comunicación Integrada (MAE-CO) y Ageing \& Communication (AgeCOM), de la Universidad de Alicante.

\section{Cómo citar el artículo:}

Martínez Sala, A. M., Barrientos-Báez, A. y Caldevilla-Domínguez, D. (2021). Fandom televisivo. Estudio de su impacto en la estrategia de comunicación en redes sociales de Netflix. Revista de Comunicación de la SEECI, 54, 57-80. http://doi.org/10.15198/seeci.2021.54.e689

\section{RESUMEN}

Las nuevas formas de consumo audiovisual dan protagonismo al fandom televisivo por el potencial que ofrece a las organizaciones del sector, como Netflix, respecto de uno de sus públicos principales, los adolescentes. Esta investigación analiza el impacto de este fenómeno en la interacción y engagement online de los seguidores de Netflix en Twitter e Instagram. Para ello se realiza un análisis de contenido cuantitativo que clasifica las publicaciones en función de dos indicadores: contenido y finalidad de las publicaciones. En lo que respecta al primero, el contenido, la

1 Alba-María Martínez-Sala: Profesora Ayudante Doctora (acreditada a Contratada Doctora por la ANECA y AVAP) en la Universidad de Alicante (UA), en el departamento de Comunicación y Psicología Social (Grado oficial y Másteres oficiales).

albamaria.martinez@ua.es 
distribución está bastante equilibrada y las publicaciones que versan sobre series de televisión generan buenos niveles de interacción y engagement online revelando la incidencia del fandom televisivo, sobre todo en la red social Instagram. Con relación al segundo, la finalidad, se observa una escasa presencia de las participativas pese a que generan mayores niveles de interacción y engagement online. Se concluye acerca de las ventajas del fandom televisivo y de una incipiente explotación del mismo que puede mejorarse dotando a las publicaciones sobre series de un carácter participativo, pero, también, se constata un correcto enfoque de la estrategia de comunicación de la marca al contemplar distintas actuaciones acordes a las características y públicos representativos de cada red social.

PALABRAS CLAVE: Adolescentes - Engagement - Estrategia de comunicación Fandom televisivo - Instagram - Netflix - Redes sociales - Series TV.

\section{ABSTRACT}

The new forms of audiovisual content consumption give an important role to the fandom television due to the potential it offers to organizations in the sector such as Netflix, regarding one of its main audiences, the teenagers. This research analyses the impact of this phenomenon on the online interaction and engagement of Netflix followers on Twitter and Instagram. To this end, a quantitative content analysis is carried out that classifies the publications based on two indicators: content and purpose of the publications. Regarding content, the first indicator, the distribution of publications is quite balanced and publications with content about television series generate good levels of interaction and online engagement. This confirms the incidence of television fandom, especially on Instagram. With regard to the purpose of the publications, the second indicator, a scarce presence of participatory publications is observed despite that these publications generate higher levels of interaction and online engagement. It concludes about the advantages of television fandom and its emerging use that can be improved by providing publications on series with a collaborative character. But it is also found that the brand's communication strategy has been correctly designed because it includes different actions depending on the different characteristics and audiences of each of the two analysed social networks.

KEY WORDS: Teenagers - Engagement - Communication Strategy - Television Fandom - Instagram - Netflix - Social Networks - TV Series.

\section{A RESISTÊNCIA DOS MEIOS DE COMUNICAÇÃO À MUDANÇA PERANTE A IMPLANTAÇÃO DAS NOVAS TECNOLOGIAS DA INFORMAÇÃO E A COMUNICAÇÃO SOCIAL}

\section{RESUMO:}

Objetivo: É responsabilidade dos meios de comunicação, como forma de serviço público, se adaptar a realidade tecnológica multimídia, para continuar desempenhando com eficácia seu trabalho informativo. Porém, qualquer processo de 
Martínez Sala, A. M., Barrientos-Báez, A. y Caldevilla-Domínguez, D. Fandom televisivo.

Estudio de su impacto en la estrategia de comunicación en redes sociales de Netflix.

mudança nas organizações, implica uma resistência inicial e um processo de adaptação das consequências e possíveis estratégias de prevenção, através de situações reais em um meio de comunicação (RTVE).

Metodologia: Revisão bibliográfica sistemática da literatura sobre a resistência às mudanças nos meios de comunicação perante a introdução de novas tecnologias e da experiência publicada sobre três cenários: digitalização de Informativos, Redação Digital Centralizada nos centros territoriais e efeitos da pandemia COVID-19.

Discussão-Resultados: São expostos resultados sobre mecanismos envolvidos na resistência às mudanças, assim como algumas características dos profissionais (trajetória profissional, experiência com novas tecnologias) e do processo (mudanças da função que impliquem sair da zona de conforto do profissional). Se apresentam os mecanismos de oposição às mudanças (greve, rejeição encoberta) e as estratégias preventivas que foram efetivas (implicação dos profissionais na esquematização do processo, eficácia dos dinamizadores ou reforços positivos na forma de incentivos).

Conclusões: $O$ profissionalismo e a quantidade de anos na função do time pode ter contribuído com a rejeição às mudanças, embora tenha garantido implantá-lo com poucos conflitos. Algumas das estratégias eficazes para superar a resistência temos os reforços positivos, a participação na criação dos profissionais e o aumento da comunicação bidirecional fundamentada nas próprias tecnologias (redes sociais).

PALAVRAS CHAVE: TICS - meios de comunicação - resistência às mudanças redatores - pandemia - Informativos - digitalização.

\section{INTRODUCCIÓN}

El desarrollo de las tecnologías de la información y la comunicación (TIC) ha provocado, especialmente entre los más jóvenes, nuevos hábitos de consumo audiovisual en los que las series de ficción cobran gran protagonismo (Marta-Lazo \& Gabelas-Barroso, 2013; Pavón-Arrizabalaga et al., 2016; Ugalde Lujambio et al., 2017). En este ámbito la combinación televisión e Internet ha provocado un cambio transcendental en la forma en la que los fans consumen contenidos y se relacionan con estos y entre ellos (Miranda-Galbe \& Cabezuelo-Lorenzo, 2018; Miranda Galbe \& Figuero Espadas, 2016; Rodríguez Pastene-Vicencio, 2017; Scolari, 2013). A este respecto, y centrándose en los adolescentes, se observa un consumo de contenidos audiovisuales individualizado a través de múltiples canales (Francisco CabezueloLorenzo et al., 2020; Ramos Méndez \& Ortega-Mohedano, 2017) con roles de género fluidos (Padilla Castillo \& Sosa Sánchez, 2018). Entre estos destacan las redes sociales para una comunicación constante entre fans (Gabriel et al., 2015) en la que los contenidos de ficción gozan de un papel relevante (Pavón-Arrizabalaga et al., 2016; Ugalde Lujambio et al., 2017).

El notable éxito del formato serie (Becattini, 2018; Francisco Cabezuelo-Lorenzo et al., 2020; Fernández-Gómez \& Martín-Quevedo, 2018a; Torregrosa-Carmona \& Rodríguez-Gómez, 2017) no ha pasado inadvertido entre las cadenas de televisión 
Martínez Sala, A. M., Barrientos-Báez, A. y Caldevilla-Domínguez, D. Fandom televisivo.

Estudio de su impacto en la estrategia de comunicación en redes sociales de Netflix.

(Cerdán Martínez, 2018) que han puesto en marcha plataformas de distribución digital de contenidos audiovisuales para dar respuesta a este nuevo contexto y consiguiente demanda (García Leiva, 2019). Entre estas cabe destacar a Netflix por tratarse de un proyecto pionero que ha sabido atender a la perfección las nuevas expectativas de la audiencia convirtiéndose en el canal de pago de mayor crecimiento en el mercado español y en uno de los principales competidores de la televisión tradicional (Fernández-Gómez \& Martín-Quevedo, 2018b; Martínez-Sala \& Prado-Jiménez, 2019; Mayorga Escalada, 2019). Netflix es el "principal impulsor de un nuevo concepto, el entretenimiento del individuo, frente al tradicional entretenimiento del hogar" (Neira, 2018, p. 69). El fenómeno observado se acrecienta entre los adolescentes, uno de los públicos principales de esta plataforma (Becattini, 2018; Gil-Quintana \& Amoros, 2020).

Los adolescentes nacieron y están creciendo en un contexto mediático marcado por la evolución de Internet y la implementación consecutiva de los modelos web 1.0, 2.0, 3.0, etc. Se trata por lo tanto de un entorno muy diversificado y eminentemente digital (Gil-Quintana \& Amoros, 2020). Su estrecha relación con las TIC llevó a Prensky (2001) a rebautizarlos como nativos digitales para distinguirlos de los individuos provenientes de la etapa analógica. Por ello los adolescentes son excelentes ejemplos de uso de las TIC, incluidas las plataformas de distribución digital de contenidos audiovisuales o plataformas de televisión in streaming (Francisco Cabezuelo-Lorenzo et al., 2020; Pavón-Arrizabalaga et al., 2016; Santamaria López et al., 2019). A este respecto, este segmento de la audiencia se caracteriza por un consumo multipantalla y en second screen que implica una exposición simultánea a contenidos digitales a través de diferentes dispositivos: portátil, smartphone, etc. (Castillo-Pomeda, 2016; Ramos Méndez \& OrtegaMohedano, 2017), y por la adopción de un rol más activo en el proceso comunicacional derivado (Atarama-Rojas \& Requena Zapata, 2018; Ugalde Lujambio et al., 2017). Este es uno de los rasgos más notables de la digitalización audiovisual (Torregrosa-Carmona \& Rodríguez-Gómez, 2017) que ha sido posible gracias a un desarrollo tecnológico al servicio de las nuevas prácticas de consumo de contenidos de las audiencias (Scolari, 2013), fundamentalmente, de las integradas por los más jóvenes ya que han sido su principal impulsor (Morimoto \& Friedland, 2011; Rubio Gil, 2010).

La industria audiovisual se enfrenta a nuevos modelos de distribución y de procesos comunicativos con sus públicos en los que debe potenciar la multidireccionalidad de la comunicación (Atarama-Rojas \& Requena Zapata, 2018; Piñeiro-Otero, 2020; Ramos Méndez \& Ortega-Mohedano, 2017). En consecuencia, Netflix y el resto de empresas del sector deben incentivar el papel de sus stakeholders como adprosumers (Monserrat-Gauchi et al., 2017; Monserrat-Gauchi \& Martínez-Sala, 2016) porque bajo el modelo web 2.0 los comentarios de los usuarios ejercen mayor influencia que los de las propias organizaciones, favoreciendo la fidelización a través de la creación de comunidades (Atarama-Rojas \& Requena Zapata, 2018; Barrientos-Bueno, 2015; Fernández-Gómez \& Martín-Quevedo, 2018b; Miranda Galbe \& Figuero Espadas, 2016; Quintas-Froufe \& González-Neira, 2014). Esto implica ciertas dificultades ya que las audiencias actuales son fieles a los 
Martínez Sala, A. M., Barrientos-Báez, A. y Caldevilla-Domínguez, D. Fandom televisivo.

Estudio de su impacto en la estrategia de comunicación en redes sociales de Netflix.

contenidos y, no tanto, a la plataforma o marca (Fernández-Gómez \& MartínQuevedo, 2018a; Muruzábal \& Grandío, 2009).

Con la llegada de Internet, y en especial del modelo web 2.0, el concepto de comunidad de marca adquiere una nueva dimensión porque los espacios y los canales de comunicación de sus miembros se multiplican exponencialmente. Tal y como señala Bellón Sánchez de la Blanca (2012), los consumidores actuales, los prosumers o adprosumers, constituyen comunidades virtuales a partir de las relaciones que establecen entre ellos y con la propia marca a través de Internet y, en especial de las redes sociales. Este es el fin último de cualquier organización que quiera sobrevivir en el mercado actual y para lograrlo es crucial plantear una estrategia de comunicación en redes sociales que favorezca las conversaciones sobre la marca con el fin de incentivar el engagement online (Armírola Garcés et al., 2020; Martínez-Sala \& Segarra-Saavedra, 2020; Monserrat-Gauchi et al., 2017; MonserratGauchi \& Martínez-Sala, 2016). Este índice es una manifestación de las relaciones que las marcas establecen con sus stakeholders y la base de sus comunidades virtuales (Monserrat-Gauchi et al., 2017; Monserrat-Gauchi \& Martínez-Sala, 2016).

A este respecto, las plataformas de distribución de contenidos audiovisuales no son una excepción ya que las redes sociales se han convertido en referentes de acceso a este tipo de contenidos (Bellón Sánchez de la Blanca, 2012; FernándezGómez \& Martín-Quevedo, 2018b; Lacalle \& Castro, 2018; Ramos Méndez \& OrtegaMohedano, 2017). Bajo el nuevo modelo de televisión, denominado "televisión social" (Herrero Subías \& Urgellés Molina, 2018; Lacalle \& Castro, 2018; Quintas-Froufe \& González-Neira, 2014), por su carácter participativo (Jenkins, 2010; Navar-Gill, 2018), estos canales han adquirido un papel fundamental en las estrategias de marketing y comunicación de las compañías de este sector (Armírola Garcés et al., 2020; Fernández-Gómez \& Martín-Quevedo, 2018b, 2018a; Navar-Gill, 2018), siendo un objetivo prioritario lograr un alto índice de engagement online (Fernández-Gómez \& Martín-Quevedo, 2018b, 2018a; Neira, 2018; Quintas-Froufe \& González-Neira, 2014).

En las redes sociales, el engagement online se mide a partir del nivel de interacción logrado, y este se expresa en términos de reacciones o "me gusta" / "no me gusta", según el canal, "compartir" y "comentar" (Atarama-Rojas \& Requena Zapata, 2018; Fernández-Gómez \& Martín-Quevedo, 2018b; Martínez-Sala \& Segarra-Saavedra, 2020; Navar-Gill, 2018). Las marcas, por lo tanto, necesitan saber cómo potenciar estas formas de interacción mediante el conjunto de variables que gestionan: frecuencia, formato, tipo de contenido, etc. Con este fin se han desarrollado numerosos estudios (Fernández-Gómez \& Martín-Quevedo, 2018b, 2018a; Martínez-Sala \& Segarra-Saavedra, 2020; Monserrat-Gauchi et al., 2017; Monserrat-Gauchi \& Martínez-Sala, 2016) que coinciden en la necesidad de partir de la premisa de que las redes sociales son conversación y que para promoverla se debe crear una comunidad de seguidores o fans identificados con la marca hasta el punto de querer convertirse en adprosumers de la misma (Fernández-Gómez \& Martín-Quevedo, 2018b; Miranda Galbe \& Figuero Espadas, 2016; Monserrat-Gauchi et al., 2017; Quintas-Froufe \& González-Neira, 2014). La creación de esa comunidad 
Martínez Sala, A. M., Barrientos-Báez, A. y Caldevilla-Domínguez, D. Fandom televisivo.

Estudio de su impacto en la estrategia de comunicación en redes sociales de Netflix.

supone el contexto idóneo para desarrollar su estrategia de comunicación a partir del establecimiento de relaciones con sus seguidores o fans (Armírola Garcés et al., 2020; Mayorga Escalada, 2019; Monserrat-Gauchi \& Martínez-Sala, 2016).

\subsection{El fandom televisivo al servicio de las marcas}

En el contexto descrito, y en el ámbito de la distribución y difusión de producciones audiovisuales, surge con fuerza la cultura fandom y, de forma específica, el fandom televisivo por el potencial comunicacional y relacional que ofrece a las organizaciones del sector como Netflix (Lacalle \& Castro, 2018; MartínezSala \& Prado-Jiménez, 2019; Torregrosa-Carmona \& Rodríguez-Gómez, 2017). La creación de una comunidad a partir del sentimiento fandom puede contribuir al éxito de sus productos mediante la generación de comentarios, publicaciones, etc., de los fans (Lacalle \& Castro, 2018). Tal y como señalan Miranda Galbe \& Figuero Espadas (2016) las aportaciones de los prosumers y adprosumers inciden directamente en la captación y fidelización de otros seguidores y, a este respecto se constata que los fans de las series de televisión, incluso los de otras series, tienen mayor predisposición a adoptar estos roles (Jost, 2014; Torregrosa-Carmona \& RodríguezGómez, 2017). Estos, reunidos en comunidades, cogeneran un saber compartido, convirtiéndose en auténticos líderes de opinión (Jenkins, 2010).

El fenómeno fan, el fandom televisivo no es algo reciente (Acevedo-Merlano, 2020; Álvarez Gandolfi, 2016) pero el modelo web 2.0 y, en especial, las redes sociales han multiplicado su visibilidad y alcance al propiciar la creación de espacios virtuales en los que sus integrantes pueden interactuar y relacionarse respecto de los contenidos televisivos como manifestación formal de pertenencia a una comunidad (Jenkins, 2010; Lacalle \& Castro, 2018). Los fans de las series de televisión recurren a las redes sociales para mantenerse informados y en contacto permanente, creando comunidades online, alrededor de sus series de televisión favoritas, en las que interactúan sin ningún tipo de restricción (Miranda Galbe \& Figuero Espadas, 2016).

Las marcas deben fomentar esta interacción e intentar participar en los procesos comunicativos que se establecen (Scolari, 2013) creando espacios propios que puedan gestionar acorde a sus objetivos empresariales pero, sobre todo, a las expectativas y deseos de sus fans (Fernández-Gómez \& Martín-Quevedo, 2018a, 2018b; Mayorga Escalada, 2019; Quintas-Froufe \& González-Neira, 2014).

Creados estos espacios, es decir sus perfiles propios en las redes sociales, la cuestión radica en cómo lograr conversación a partir de la conversión del consumidor en prosumer o adprosumer. Ciertamente es más sencillo lograrlo cuando existe un interés previo por el producto o servicio como ocurre con los fans de las series de televisión. En su caso, además, las incógnitas, la falta de respuesta a algunas preguntas planteadas por las series, o simplemente el deseo de manifestar su visión, son el principal motivo por el que se lanzan a producir y difundir sus propios contenidos. De este modo sus creaciones, los fanmades se presentan como un complemento que contribuye a la comprensión y difusión de la serie de televisión 
Martínez Sala, A. M., Barrientos-Báez, A. y Caldevilla-Domínguez, D. Fandom televisivo.

Estudio de su impacto en la estrategia de comunicación en redes sociales de Netflix.

(Miranda Galbe \& Figuero Espadas, 2016; Torregrosa-Carmona \& Rodríguez-Gómez, 2017).

En función de lo expuesto se justifica esta investigación fundamentada en la necesidad de las organizaciones de fomentar la interacción por parte de los usuarios de las redes sociales, es decir, de activar sus roles como prosumers y adoprosumers, para lograr un nivel óptimo de engagement online. Esto obliga a profesionales y académicos a reconsiderar la conceptualización del engagement, y de las fórmulas para generarlo (Martínez-Sala \& Segarra-Saavedra, 2020) porque bajo el modelo web 2.0 y, para los nativos digitales, todo gira en torno a participar y compartir (Fernández-Gómez \& Martín-Quevedo, 2018b).

\section{OBJETIVOS}

Dada la relevancia de la audiencia social (González Neira \& Quintas Froufe, 2016; Herrero Subías \& Urgellés Molina, 2018; Lacalle \& Castro, 2018) en la construcción y desarrollo de las marcas, las organizaciones del sector audiovisual, en general, y Netflix en particular, deben desarrollar estrategias de marketing y comunicación digital que integren las redes sociales de tal forma que se propicie una comunicación de carácter multidireccional promovida por la marca y por los propios seguidores convertidos en prosumers y adprosumers, y estimulada por los textos televisivos (Fernández-Gómez \& Martín-Quevedo, 2018b, 2018a; Herrero Subías \& Urgellés Molina, 2018; Lacalle \& Castro, 2018; Mayorga Escalada, 2019). Ello conlleva altos índices de engagement online. En este sentido, el contexto actual presenta dificultades respecto del logro de la implicación de los seguidores (Fernández-Gómez \& Martín-Quevedo, 2018b, 2018a; Herrero Subías \& Urgellés Molina, 2018) pero también oportunidades y ventajas relacionadas con las características de la propia audiencia y de las redes sociales. Efectivamente el fandom televisivo representa para Netflix, y su sector, a un público objetivo predispuesto a convertirse en prosumer y adprosumer (Torregrosa-Carmona \& Rodríguez-Gómez, 2017) y este estudio se centra, precisamente, en verificar el potencial de este fenómeno en dos redes sociales: Twitter e Instagram.

Así mismo se parte de la premisa de que los adolescentes, principales seguidores del fandom televisivo, conforman un mercado de enormes posibilidades comunicacionales y relacionales para las marcas por su comportamiento natural en las redes sociales (Atarama-Rojas \& Requena Zapata, 2018; Ugalde Lujambio et al., 2017). Respecto de estos canales, se ha escogido Twitter por ser el más utilizada con relación al consumo multipantalla (Harrington, 2014; Segado-Boj et al., 2015; Torregrosa-Carmona \& Rodríguez-Gómez, 2017) y para la difusión de fanmade (Marcos, 2015; Torregrosa-Carmona \& Rodríguez-Gómez, 2017). Además, el modelo de amistad dirigida en el que se fundamenta (Marwick \& Boyd, 2011b, 2011a), implica, en el sector objeto de este estudio, que los fans lo usen para informarse acerca de las series y compartir y comentar estos contenidos sin esperar una continuidad a la comunicación que han iniciado (Navar-Gill, 2018; TorregrosaCarmona \& Rodríguez-Gómez, 2017), focalizando la consecución del engagement en los contenidos. Esto facilita la fidelización de la audiencia y el engagement online 
Martínez Sala, A. M., Barrientos-Báez, A. y Caldevilla-Domínguez, D. Fandom televisivo.

Estudio de su impacto en la estrategia de comunicación en redes sociales de Netflix.

(Herrero Subías \& Urgellés Molina, 2018) ya que su gestión es controlable por las marcas. La inclusión de Instagram se debe a que el público principal objeto de esta investigación: los adolescentes, un segmento crucial para medir el efecto del fandom televisivo (Pavón-Arrizabalaga et al., 2016; Ugalde Lujambio et al., 2017), son además los principales consumidores de esta red social (Gil-Quintana \& Amoros, 2020). La comparación entre ambas redes sociales contribuye al análisis de la incidencia del fandom televisivo en la medida en la que Twitter es una de las redes sociales preferidas con relación al consumo de productos audiovisuales (Harrington, 2014; Segado-Boj et al., 2015; Torregrosa-Carmona \& RodríguezGómez, 2017) e Instagram, no solo destaca en este mismo ámbito (Neira, 2015; Ramos Méndez \& Ortega-Mohedano, 2017; Torregrosa-Carmona \& RodríguezGómez, 2017) sino que además concentra en mayor medida al público adolescente (Gil-Quintana \& Amoros, 2020). Se espera, en consecuencia, una mayor incidencia del fandom televisivo sobre la interacción de los usuarios en Instagram que en Twitter.

Los argumentos y hechos constatados justifican este estudio que persigue dar respuesta a las siguientes preguntas de investigación:

P.I.1: ¿La marca Netflix ha considerado e incorporado el fenómeno fandom televisivo en su estrategia de comunicación en las redes sociales Twitter e Instagram con el fin de aprovechar el potencial comunicacional y relacional que ofrecen los adolescentes respecto del logro del engagement online?

P.I.2: ¿El fandom televisivo incide de forma diferente en el nivel de engagement online según la red social y sus respectivas audiencias?

En consecuencia, el objetivo principal de esta investigación radica en el análisis del impacto del fandom televisivo en la interacción generada por los usuarios de los perfiles de Netflix en Twitter e Instagram, teniendo en cuenta el papel relevante de los adolescentes a este respecto, así como su importancia para las marcas (AtaramaRojas \& Requena Zapata, 2018; Gil-Quintana \& Amoros, 2020; Pavón-Arrizabalaga et al., 2016; Ugalde Lujambio et al., 2017).

A partir de los resultados se podrá concluir acerca de las ventajas de considerar este fenómeno en el diseño y desarrollo de las estrategias de marketing y comunicación digital de la marca Netflix respecto de favorecer el engagement online.

Para dar respuesta a las preguntas de investigación y, en atención al objetivo principal, se plantean los siguientes objetivos específicos:

O1: Determinar y comparar la presencia de Netflix (su perfil, sus seguidores, etc.) en Twitter e Instagram.

O2: Clasificación y comparación de las publicaciones, en Twitter e Instagram, según contenido (series vs. no series, series originales vs. series no originales) y finalidad principal (objetivo comercial o participativo).

03: Evaluar la interacción y engagement online que generan las distintas categorías de publicaciones en Twitter e Instagram y compararlos. 
Martínez Sala, A. M., Barrientos-Báez, A. y Caldevilla-Domínguez, D. Fandom televisivo.

Estudio de su impacto en la estrategia de comunicación en redes sociales de Netflix.

Cabe señalar que la clasificación de las publicaciones no se limita a la consideración del fandom televisivo (contenido) sino que se contempla también la finalidad por su incidencia en los niveles de interacción (Fernández-Gómez \& MartínQuevedo, 2018b; Monserrat-Gauchi et al., 2017; Monserrat-Gauchi \& Martínez-Sala, 2016).

En conjunto, el estudio que se plantea indaga acerca de cómo las compañías de distribución de contenidos audiovisuales pueden aprovechar el proceso de transformación que está experimentando su mercado, liderado por los adolescentes (Ramos Méndez \& Ortega-Mohedano, 2017), atendiendo a sus expectativas y deseos. La incorporación del fandom televisivo en la estrategia de comunicación parece ser una clara respuesta a ello, al ser un movimiento característico de esta generación. Del mismo modo, el uso de las redes sociales con carácter multidireccional también es una buena medida ya que atiende sus necesidades y prácticas comunicativas (Lacalle \& Castro, 2018; Torregrosa-Carmona \& RodríguezGómez, 2017)

\section{METODOLOGÍA}

Las preguntas de investigación y objetivos planteados se abordan mediante un análisis de contenido cuantitativo de las publicaciones de la marca Netflix y de la interacción generada en sus perfiles corporativos en Twitter e Instagram desde un enfoque metodológico exploratorio (Batthyány \& Cabrera, 2011). Este tipo de análisis es adecuado por la objetividad que ofrece en los resultados y su eficiencia en el análisis de contenidos comunicacionales (Berelson, 1952), incluidos los de las redes sociales. Prueba de ello son los numerosos estudios que han recurrido a esta técnica en este mismo ámbito, con relación a empresas de todo tipo, incluidas las plataformas de distribución de contenidos audiovisuales (Atarama-Rojas \& Requena Zapata, 2018; Fernández-Gómez \& Martín-Quevedo, 2018b, 2018a; González Neira \& Quintas Froufe, 2016; Lacalle \& Castro, 2018; Miranda Galbe \& Figuero Espadas, 2016).

La ficha de análisis se diseñó a partir de los estudios revisados sobre plataformas de distribución de contenidos audiovisuales y sobre fandom televisivo (Becattini, 2018; Fernández-Gómez \& Martín-Quevedo, 2018b, 2018a; Herrero Subías \& Urgellés Molina, 2018; Martínez-Sala \& Prado-Jiménez, 2019; Mayorga Escalada, 2019; Segado-Boj et al., 2015). La propuesta inicial fue evaluada y validada por dos investigadoras académicas de acreditada trayectoria en el ámbito de la comunicación digital y por una prestigiosa empresa especializada en marketing digital. El análisis fue realizado por una única persona a partir de la ficha de análisis resultante (Tabla 1). Esta consta de tres apartados: presencia, tipología de las publicaciones e interacción. La tipología contempla dos subapartados: contenido y finalidad. El primero se ocupa del potencial del fandom televisivo y distingue las publicaciones relativas a series del resto. Además, dentro de las primeras se diferencia las series propias de la cadena de las producidas por otras plataformas dado que, como se ha constatado, incluso los fans de otras series tienen mayor predisposición a interactuar (Torregrosa-Carmona \& Rodríguez-Gómez, 2017). Así mismo, esta 
Martínez Sala, A. M., Barrientos-Báez, A. y Caldevilla-Domínguez, D. Fandom televisivo.

Estudio de su impacto en la estrategia de comunicación en redes sociales de Netflix.

distinción contribuye al logro del objetivo principal de esta investigación: dotar a la marca Netflix de las herramientas necesarias para atender las expectativas del público adolescente al tiempo que logra sus propios objetivos.

La dimensión relativa a la finalidad se construye a partir del tipo de comunicación que se potencia, la unidireccional y la bidireccional o multidireccional, distinguiendo entre las publicaciones comerciales y participativas respectivamente. Esta categorización se incluye por la importancia que esta característica tiene para los usuarios, máxime en el caso de los adolescentes, siendo crucial para la generación de conversación (Atarama-Rojas \& Requena Zapata, 2018; Ugalde Lujambio et al., 2017).

En el último apartado, el relativo a la interacción, se cuantifica el número de "me gusta", de "retweets" y de comentarios. Los datos resultantes permiten calcular el engagement online (Martínez-Sala \& Segarra-Saavedra, 2020).

\section{Tabla 1. Ficha de análisis}

\begin{tabular}{|c|c|}
\hline \multicolumn{2}{|l|}{ PRESENCIA } \\
\hline url & Enlace al perfil de la marca en Twitter e Instagram \\
\hline Total publicaciones & $\begin{array}{l}\text { Número total de publicaciones difundidas por la marca al } \\
\text { finalizar el periodo de análisis. }\end{array}$ \\
\hline Siguiendo & Número de perfiles seguidos al finalizar el periodo de análisis. \\
\hline Seguidores & Número de seguidores al finalizar el periodo de análisis. \\
\hline Total "me gusta" & Número total de "me gusta" al finalizar el periodo de análisis. \\
\hline \multicolumn{2}{|l|}{ TIPOLOGÍA CONTENIDO } \\
\hline Publicaciones sobre series & $\begin{array}{l}\text { Número total de publicaciones con contenidos relativos a } \\
\text { series de televisión (tanto originales o propias de la compañía, } \\
\text { como ajenas). }\end{array}$ \\
\hline ... sobre series originales & $\begin{array}{l}\text { Número total de publicaciones con contenidos relativos a } \\
\text { series de televisión originales, o propias, de la compañía. }\end{array}$ \\
\hline ... sobre series no originales & $\begin{array}{l}\text { Número total de publicaciones con contenidos relativos a } \\
\text { series de producción ajena a la compañía. }\end{array}$ \\
\hline $\begin{array}{l}\text { Publicaciones sobre otros (no } \\
\text { series) }\end{array}$ & $\begin{array}{l}\text { Número total de publicaciones con contenidos relativos a } \\
\text { cualquier temática ajena a las series de televisión. }\end{array}$ \\
\hline \multicolumn{2}{|l|}{ TIPOLOGÍA FINALIDAD } \\
\hline Comercial & $\begin{array}{l}\text { Número total de publicaciones con finalidad comercial } \\
\text { (promoción, autopromoción, etc.) }\end{array}$ \\
\hline Participativa & $\begin{array}{l}\text { Número total de publicaciones con finalidad participativa } \\
\text { (encuestas, preguntas, etc.) }\end{array}$ \\
\hline Otros & Número total del resto de publicaciones. \\
\hline \multicolumn{2}{|l|}{ INTERACCIÓN } \\
\hline "me gusta" & Número total de "me gusta" por publicación. \\
\hline "Retweet"o "compartir"* & Número total de veces que ha sido compartida la publicación. \\
\hline "comentar" & Número total de comentarios que ha generado la publicación. \\
\hline Engagement online & $\frac{\text { "me gusta" + "retweets"* + "comentar" }}{\text { No de publicaciones }} \times 100$ \\
\hline
\end{tabular}

${ }^{2}$ Los "retweets" solo se contemplan en el caso de Twitter ya que los datos relativos a la opción equivalente en Instagram: "compartir" no son visibles. 
Martínez Sala, A. M., Barrientos-Báez, A. y Caldevilla-Domínguez, D. Fandom televisivo.

Estudio de su impacto en la estrategia de comunicación en redes sociales de Netflix.

Fuente: Elaboración propia.

Nota: Instagram no proporciona información relativa al número de veces que ha sido compartida la publicación por lo que este dato no se considera individualmente ni para el cálculo del engagement online tal y como ya hicieron Atarama-Rojas y Requena Zapata (2018).

El periodo analizado comprende 1 año (19 marzo 2018 - 19 marzo 2019). Durante este, Netflix publicó 1.087 tweets. Su análisis, así como el de la interacción generada se realiza aplicando el algoritmo Twitter Decahose y eligiendo, por consiguiente, una muestra del 10\% del total (Brantner \& Rodriguez-Amat, 2016; Fernández-Gómez \& Martín-Quevedo, 2018b; Martínez-Sala \& Prado-Jiménez, 2019). En el caso de Instagram se analiza la misma cantidad de publicaciones para garantizar la objetividad de la comparativa.

\section{DISCUSIÓN}

\subsection{Presencia y tipología de las publicaciones}

Con relación a la P.I.1, se analizan la presencia de Netflix en ambas redes sociales (01) y sus publicaciones, para clasificarlas según contenido y finalidad principal (O2) (Tabla 2).

Tabla 2. Presencia y tipología de la comunicación de Netflix en Twitter e Instagram

\begin{tabular}{|c|c|c|}
\hline PRESENCIA & TWITTER & INSTAGRAM \\
\hline url & https://twitter.com/NetflixES & https://www.instagram.com/netflixes/ \\
\hline Total publicaciones & $12,3 \mathrm{mil}$ & 937 \\
\hline Siquiendo & 294 & 165 \\
\hline Seguidores & 760.000 & $1,2 \mathrm{mil}$ \\
\hline "Me gusta" & 3.207 & - \\
\hline \multicolumn{3}{|c|}{ TIPOLOGÍA CONTENIDO } \\
\hline Publicaciones sobre series & 55 & 63 \\
\hline ...sobre series originales & 49 & 61 \\
\hline ...sobre series no originales & 6 & 2 \\
\hline Publicaciones sobre otros (no series) & 45 & 37 \\
\hline \multicolumn{3}{|c|}{ TIPOLOGÍA FINALIDAD } \\
\hline Comercial & 82 & 72 \\
\hline Participativa & 15 & 20 \\
\hline Otros & 3 & 8 \\
\hline
\end{tabular}

Fuente: Elaboración propia.

En Twitter, Netflix tiene 12,3 mil publicaciones y un total de 760.000 seguidores que han generado 3.207 "me gusta". Instagram, con un número muy inferior de publicaciones, cuenta, sin embargo, con una comunidad mayor (1,2 mil seguidores).

De la muestra de publicaciones analizadas, en Twitter aproximadamente, la mitad (55) son sobre series, y en particular, de producción propia (49), mientras que el 
resto (45) se centran en otro tipo de contenidos como estrenos de documentales o películas. No obstante, se ha podido comprobar que para este tipo de contenidos audiovisuales tienen además otro perfil específico: @NetflixPelis.

Respecto de la finalidad de las publicaciones, predominan las de carácter comercial (82) muy por delante de las que invitan a participar (15). De hecho, cabe señalar que no se ha publicado ningún tweet sobre series (original o no) que sea participativo.

Se muestra a continuación un ejemplo del tipo de tweet más frecuente.

Netflix España @NetflixES * 6 mar.

Esto es real. Esto es mágico. Esto es 'Cien Años de Soledad'. La serie llega próximamente.

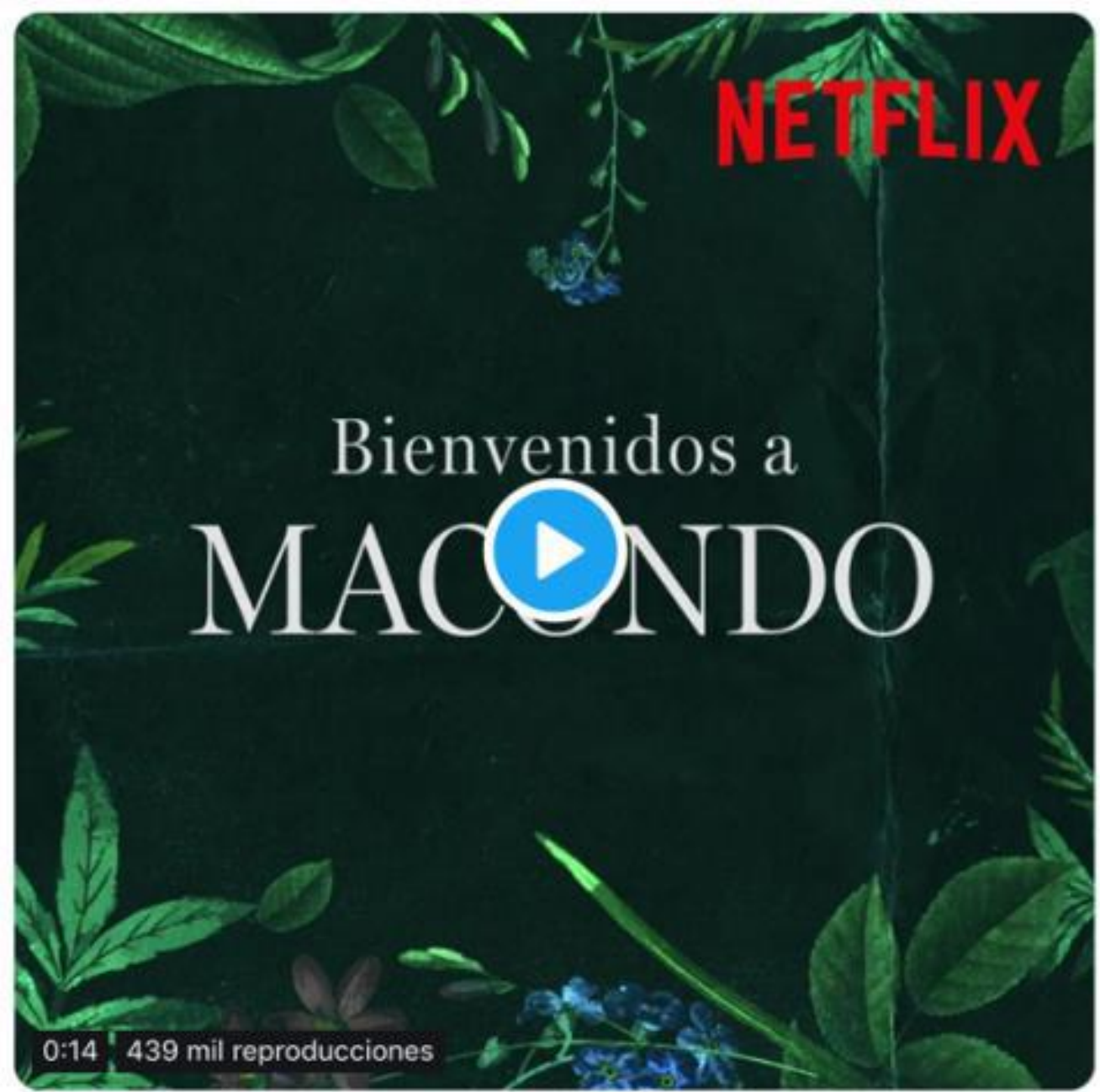

Q $426 \quad$ † $3,5 \mathrm{~K} \quad \bigcirc \quad 11 \mathrm{~K}$

Imagen 1. Tweet sobre serie original con finalidad comercial Fuente: @NetflixES, Twitter (2019, 6 marzo) 
En lo que respecta a Instagram, la diferencia entre el total de publicaciones sobre series (63) y el resto (37) es mayor y se mantiene el predominio de aquellas que versan sobre series originales (61). Igualmente se observan diferencias en lo que respecta a la proporción de publicaciones participativas (20) respecto de las comerciales (72) ya que hay un mayor número de participativas, aunque también predominan las comerciales.

\subsection{Interacción y engagement online}

Identificadas y catalogadas las publicaciones se concreta el grado de interacción logrado por cada una de ellas (Tabla 3). Esta información relativa al O3 sirve para dar respuesta a la P.I.2. Para ello, y con el fin de poder realizar comparaciones objetivas se han complementado los resultados totales con los promedios por publicación (Tabla 4).

Tabla 3. Interacción y engagement online, según tipología, en Twitter (TW) e Instagram (INST) (Total)

\begin{tabular}{|c|c|c|c|c|c|c|c|c|}
\hline & \multicolumn{2}{|c|}{ "Me gusta" } & \multicolumn{2}{|c|}{ "Retweet" } & \multicolumn{2}{|c|}{ "Comentar" } & \multicolumn{2}{|c|}{ Engagement online } \\
\hline & TW & INST & TW & INST & TW & INST & TW & INST \\
\hline $\begin{array}{l}\text { TOTAL PUBLICACIONES } \\
(000)\end{array}$ & 155.878 & 3.824 .363 & 36.125 & - & 6.533 & 65.960 & 198.536 & 3.890 .323 \\
\hline \multicolumn{9}{|c|}{ TIPOLOGÍA CONTENIDO (\% sobre el total) } \\
\hline Publicaciones sobre series & $43,63 \%$ & & $46,5 \%$ & - & $25,06 \%$ & & $79,1 \%$ & $96,3 \%$ \\
\hline ...sobre series originales & $34,98 \%$ & $57 \%$ & $38,5 \%$ & - & $19,36 \%$ & $49,0 \%$ & $71,6 \%$ & $93,6 \%$ \\
\hline ...sobre series no originales & $8,64 \%$ & $4 \%$ & $8,0 \%$ & - & $1,56 \%$ & $5,3 \%$ & $138,2 \%$ & $178,3 \%$ \\
\hline $\begin{array}{l}\text { Publicaciones sobre otros } \\
\text { (no series) }\end{array}$ & $56,37 \%$ & $39 \%$ & $53,5 \%$ & - & $4 \%$ & $45,7 \%$ & $125,5 \%$ & $106,8 \%$ \\
\hline \multicolumn{9}{|c|}{ TIPOLOGÍA FINALIDAD (\% sobre el total) } \\
\hline Comercial & $60,3 \%$ & $67 \%$ & $62,3 \%$ & - & $34,21 \%$ & $82,7 \%$ & $72,9 \%$ & $92,8 \%$ \\
\hline Participativa & $36,2 \%$ & $28 \%$ & $34,2 \%$ & - & $63,97 \%$ & $14,6 \%$ & $245,2 \%$ & $140,7 \%$ \\
\hline Otros & $3,5 \%$ & $5 \%$ & $3,6 \%$ & - & $1,82 \%$ & $2,7 \%$ & $114,3 \%$ & $62,9 \%$ \\
\hline
\end{tabular}

Fuente: Elaboración propia.

Acorde a lo expuesto en la tabla 3, en Twitter la opción de interacción más utilizada es "me gusta" seguida de "retweet" y, por último, "comentar". Esta tendencia observada respecto del total de tweets se mantiene en todas las categorías. Lo mismo ocurre en Instagram.

Por tipología en Twitter, son los tweets sobre otros contenidos (no series) y los de carácter comercial los que contabilizan los mayores totales en todas las opciones de interacción, excepto en el caso de "comentar" que, bajo el indicador relativo a finalidad, se produce en mayor medida en las publicaciones participativas (63,97\%). En Instagram, sin embargo, son las publicaciones sobre series las que contabilizan el mayor número de "me gusta" y de comentarios debido, fundamentalmente, a las 
relativas a series de producción propia. En lo concerniente a la finalidad, son las publicaciones de carácter comercial las que lideran ambos rankings, también el de los comentarios a diferencia de lo que ocurre en Twitter. Pese a esto, en términos de engagement online son las publicaciones participativas las que logran mejores resultados $(140,7 \%)$ junto a las que versan sobre series, pero, en este caso, de producción ajena $(178,3 \%)$, contrariamente a los resultados obtenidos individualmente por cada una de las dos opciones de interacción.

En lo concerniente a Twitter, a diferencia de lo descrito a nivel de las opciones de interacción, los resultados relativos al engagement online evidencian que son los tweets sobre series no originales los que mayor repercusión han tenido entre la audiencia social $(138,2 \%)$. De igual modo son los tweets participativos los que mayor índice de engagement online han logrado $(245,2 \%)$, en línea con lo observado con relación al número de comentarios (63,97\%).

Los resultados promedio/tweet confirman, en gran parte, lo observado en términos de engagement online (Tabla 4).

Tabla 4. Interacción y engagement online, según tipología, en Twitter (TW) e Instagram (INST) (Promedio/Publicación)

\begin{tabular}{|c|c|c|c|c|c|c|}
\hline & \multicolumn{2}{|c|}{ "Me gusta" } & \multicolumn{2}{|c|}{ "Retweet" } & \multicolumn{2}{|c|}{ "Comentar" } \\
\hline & TW & INST & TW & INST & TW & INST \\
\hline TOTAL PUBLICACIONES (000) & 1.558 & $38.243,6$ & 361,25 & - & 65,33 & 659,6 \\
\hline \multicolumn{7}{|c|}{ TIPOLOGÍA CONTENIDO (\% sobre el total) } \\
\hline Publicaciones sobre series & $79,4 \%$ & $96,5 \%$ & $84,5 \%$ & - & $45,6 \%$ & $86,2 \%$ \\
\hline ..sobre series originales & $71,4 \%$ & $93,9 \%$ & $78,5 \%$ & - & $39,5 \%$ & $80,3 \%$ \\
\hline ...sobre series no originales & $144,1 \%$ & $176,8 \%$ & $133,1 \%$ & - & $26,0 \%$ & $266,2 \%$ \\
\hline Publicaciones sobre otros (no series) & $125,3 \%$ & $106,0 \%$ & $119,0 \%$ & - & $175,7 \%$ & $123,5 \%$ \\
\hline \multicolumn{7}{|c|}{ TIPOLOGÍA FINALIDAD (\% sobre el total) } \\
\hline Comercial & $73,6 \%$ & $3,4 \%$ & $75,9 \%$ & - & $41,7 \%$ & $4,7 \%$ \\
\hline Participativa & $241,8 \%$ & $141,9 \%$ & $227,7 \%$ & - & $426,5 \%$ & $72,9 \%$ \\
\hline Otros & $115,5 \%$ & $1,8 \%$ & $119,2 \%$ & - & $60,7 \%$ & $2,3 \%$ \\
\hline
\end{tabular}

Fuente: Elaboración propia.

Los datos expuestos en la tabla 4 muestran que los tweets sobre series no originales son los que logran los mejores promedios/publicación de "me gusta" y "Retweet", sin embargo, a nivel de "comentar" son los tweets sobre otros contenidos los que más interés despiertan $(175,7 \%))$. En este sentido, se mantiene lo observado en términos globales (Tabla 3) solo respecto de las opciones de interacción "comentar", mientras que en lo relativo a "me gusta" y "retweets" se confirma lo evidenciado por el índice de engagement online. En esta línea se constata que la publicación con mayor número de comentarios es, precisamente, una publicación sobre otros contenidos de carácter participativo (Imagen 2). 
Martínez Sala, A. M., Barrientos-Báez, A. y Caldevilla-Domínguez, D. Fandom televisivo. Estudio de su impacto en la estrategia de comunicación en redes sociales de Netflix.

Netflix España @NetflixES 25 feb.

Si el mundo se acabase en 8 días, ¿cuál sería la última serie que verías?

\section{3,1K † $336 \quad$ ○,9K}

Imagen 2. Tweet con mayor nivel de interacción ("comentar")

Fuente: @NetflixES, Twitter (2019, 25 febrero)

Al respecto de la finalidad son, de nuevo, las publicaciones de carácter participativo, como la anterior (Imagen 2), las que logran el promedio más alto en las tres opciones de interacción, en línea con lo observado a nivel de "comentar" y del engagement online (Tabla 3). En Instagram se mantiene el liderazgo de las publicaciones sobre series, pero en este caso de producción ajena, confirmando lo observado a nivel de engagement online. $\mathrm{Y}$, al igual que en Twitter, son las publicaciones de carácter participativo las que mejores resultados obtienen en términos de interacción tal y como se muestra en la imagen 3.

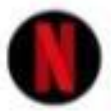

Netflix España

QNetflixES

Hola profesores de España: ¿podéis dejar de poner exámenes cuando subimos series?

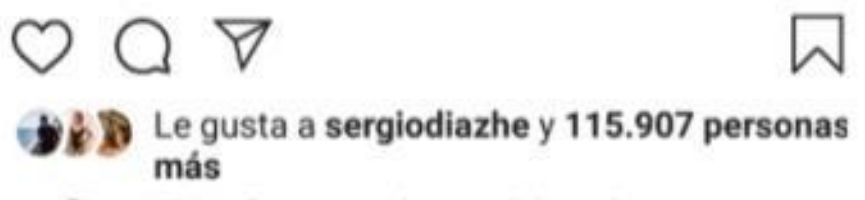

netflixes El Profesor jamás pondría exámenes en

esas fechas.

Ver los 1.352 comentarios 
Martínez Sala, A. M., Barrientos-Báez, A. y Caldevilla-Domínguez, D. Fandom televisivo.

Estudio de su impacto en la estrategia de comunicación en redes sociales de Netflix.

Imagen 3. Publicación con mayor nivel de interacción ("me gusta")

Fuente: @NetflixES, Instagram (2019, 25 enero)

Así mismo, cabe señalar que en Instagram sí hay publicaciones sobre series de carácter participativo. Concretamente hay 9 , de las que 8 son sobre series de producción propia.

\section{CONCLUSIONES}

Las compañías de distribución de contenidos audiovisuales deben fomentar que el fan interactúe en todas las plataformas, especialmente en las digitales (Francisco Cabezuelo-Lorenzo et al., 2020; Miranda Galbe \& Figuero Espadas, 2016) donde destacan las redes sociales. A este respecto existe un gran interés profesional y académico por la incidencia que tiene la actividad de la audiencia social (Herrero Subías \& Urgellés Molina, 2018; Lacalle \& Castro, 2018) en estos canales en el desarrollo y construcción de las marcas (Monserrat-Gauchi \& Martínez-Sala, 2016; Navar-Gill, 2018). Centrándose en estos, las investigaciones revisadas coinciden al respecto de la necesidad de tener una presencia constante basada en el establecimiento de una comunicación multidireccional, (González Neira \& Quintas Froufe, 2016). Esto requiere de la consecución del engagement online y ahí radica la principal dificultad para las marcas porque se enfrentan a unos consumidores cada vez más participativos en las redes sociales pero también más selectivos (QuintasFroufe \& González-Neira, 2014).

Atender esta realidad exige de un conocimiento profundo de las audiencias, de sus características, con el fin de convertirlas en ventajas. De este modo, el fandom televisivo se revela como una oportunidad para que marcas, como Netflix, logren engagement online. Los fans televisivos conforman comunidades en las que comentan, conversan, etc., sobre contenidos mediáticos por los que comparten un interés común (Atarama-Rojas \& Requena Zapata, 2018; Jenkins, 2010). Las marcas de esta industria pueden aprovechar este fenómeno potenciando que sus integrantes actúen como prosumers y adprosumers. Para ello se debe, primero, constatar que efectivamente favorece el engagement online, y en su caso, determinar cómo explotarlo.

En este sentido, se ha desarrollado esta investigación que, en primer lugar, ha confirmado cierta consideración del fandom televisivo en la estrategia de comunicación digital de Netflix en Twitter e Instagram (P.I.1). Aproximadamente la mitad de las publicaciones son sobre series de televisión, aunque sorprende el elevado número de publicaciones sobre otros contenidos. Estas publicaciones suelen estar orientadas a estimular la participación (encuestas, saludo, etc.), pero también, a la promoción de la plataforma pese a que los usuarios conectan más fácilmente con contenidos específicos que con la marca (Fernández-Gómez \& Martín-Quevedo, 2018b). Los resultados comprobados en Twitter se mantienen en Instagram, aunque en esta red social la proporción de publicaciones sobre series es mayor. 
Martínez Sala, A. M., Barrientos-Báez, A. y Caldevilla-Domínguez, D. Fandom televisivo.

Estudio de su impacto en la estrategia de comunicación en redes sociales de Netflix.

Una vez identificadas y catalogadas las publicaciones se analiza la interacción generada con el fin de responder a la P.I.2 sobre la incidencia del fandom televisivo en el nivel de engagement online. Los resultados han revelado el potencial comunicacional y relacional del fandom televisivo en ambas redes sociales y, en especial, en Instagram. En Twitter, aunque a nivel global, son los tweets sobre otros (no series) los que logran los máximos niveles de interacción, el índice de engagement online más elevado lo ostentan los tweets sobre series, concretamente de series no originales. Además, en función de los promedios/publicación son también este tipo de tweets los que mayor número de "me gusta" y "retweet" logran, a diferencia de los resultados obtenidos en este mismo ámbito por Fernández-Gómez \& Martín-Quevedo (2018b). En lo concerniente a las publicaciones sobre otros contenidos, estas lideran el promedio de comentarios, cuestión que se explica a tenor de la finalidad de las publicaciones.

El criterio relativo a la finalidad de las publicaciones se consideró por su relevancia en los niveles de interacción (Monserrat-Gauchi et al., 2017; Monserrat-Gauchi \& Martínez-Sala, 2016) y con el fin de discriminar los efectos del contenido. En este ámbito se confirma que las publicaciones participativas lideran los niveles de interacción por tweet, pese a ser las menos frecuentes. Su escaso número influye en el cómputo global de las tres opciones de interacción donde son superadas por las comerciales a nivel de "me gusta" y "compartir", en el caso de Twitter, y de "me gusta" y "comentar", en el de Instagram. En esta red social la influencia del fandom televisivo en los niveles de interacción y en el índice de engagement online es más notoria tanto con relación a los totales como a los promedios/publicación. En este canal son las publicaciones sobre series propias las que mejores resultados obtienen en términos de "me gusta" y "comentar", y las de producción ajena en el ámbito del engagement online, al igual que ocurre respecto de los promedios/publicación. En cuanto a la finalidad, las participativas consiguen un mejor nivel de engagement online en el ámbito de los totales, y de "me gusta" y comentarios, en el de los promedios/publicación. Las diversas aplicaciones facilitadoras pueden clasificarse con facilidad en función de su finalidad y servicio que ofertan (Barrientos-Báez et al., 2017).

Se confirma la incidencia del fandom televisivo en la interacción generada en el perfil de la marca en Twitter a propósito de producciones ajenas a la cadena. Esto revela una estrategia basada en contenidos relativos a producciones de alta repercusión para atraer y captar usuarios incentivando el crecimiento de la comunidad en torno a la marca (Mayorga Escalada, 2019). Pese a esto, en función de los resultados relativos a la finalidad de las publicaciones, se concluye que se puede mejorar la estrategia de comunicación incentivando el carácter multidireccional de la comunicación en las publicaciones sobre series, así como incrementando el número de publicaciones de este tipo. En Instagram la consideración del fandom televisivo es más evidente, logrando mejores resultados y mostrando que la plataforma es consciente y explota el potencial de este fenómeno entre los adolescentes, público principal de la plataforma. No obstante, como en el caso de Twitter cabe mejorarlos dotando de finalidad participativa a las publicaciones sobre series, en mayor medida. 
Martínez Sala, A. M., Barrientos-Báez, A. y Caldevilla-Domínguez, D. Fandom televisivo.

Estudio de su impacto en la estrategia de comunicación en redes sociales de Netflix.

Las diferencias observadas son otro rasgo positivo a destacar respecto de la estrategia de comunicación digital de Netflix ya que revelan la consideración de las características de cada red social, así como de las expectativas de sus respectivos públicos.

En el contexto de la televisión social, las redes sociales deben ser integradas por las marcas del sector en sus estrategias de marketing y comunicación digital (Harrington et al., 2013; Herrero Subías \& Urgellés Molina, 2018; Navar-Gill, 2018). Los contenidos vertidos en estos espacios contribuyen a la creación y desarrollo de las marcas que, en el momento actual, están en manos de los usuarios, por ello es una prioridad que sus responsables incentiven y monitoricen estos canales y las conversaciones que en ellos se generan, siendo crucial el estudio de sus detonantes (Fernández-Gómez \& Martín-Quevedo, 2018b). Entre estos, esta investigación se centra en el fandom televisivo, confirmando su potencial, pero también una explotación incipiente, sobre todo en Twitter, resultando una interesante aportación tanto para el ámbito académico como para el profesional que radica en el potencial del séptimo arte respecto del establecimiento de una cultura corporativa en nuestra acción profesional (F. Cabezuelo-Lorenzo et al., 2021). Así mismo, las numerosas ventajas del fenómeno fandom televisivo plantean nuevas líneas de investigación que subrayan su interés. A este respecto, se plantea ampliar la muestra de publicaciones y redes sociales, así como de variables a analizar para identificar otros posibles factores.

\section{REFERENCIAS}

Acevedo-Merlano, Á. (2020). El animé como lienzo para analizar las tensiones entre prácticas epistémicas ancestrales y tecnocientíficas. Utopía y Praxis Latinoamericana. Revista Internacional de Filosofía y Teoría Social, 25(89), 211226.

Álvarez Gandolfi, F. (2016). Cibercultura Otaku, un análisis interdiscursivo de identidades fan puestas en escena en grupos de Facebook. Perspectivas de La Comunicación, 9(2), 31-57.

Armírola Garcés, L. P., García Nieto, M. T., \& Romero González, G. C. (2020). La comunicación digital en las micro y pequeñas empresas: El caso del sector cultural del departamento colombiano de Bolívar. Revista de Comunicación de La SEECI, 52, 149-169. http://doi.org/10.15198/seeci.2020.52.149-169

Atarama-Rojas, T., \& Requena Zapata, S. (2018). Narrativa Transmedia: Análisis de la participación de la audiencia en la serie 13 reasons why para la aproximación al tema del suicidio. Fonseca, Journal of Communication, 17, 193-213. https://doi.org/10.14201/fjc201817193213 
Martínez Sala, A. M., Barrientos-Báez, A. y Caldevilla-Domínguez, D. Fandom televisivo.

Estudio de su impacto en la estrategia de comunicación en redes sociales de Netflix.

Barrientos-Bueno, M. (2015). Expansión narrativa de Víctor Ros: Transmedia storytelling en twitter. Opcion, 31(Special Issue 3), 161-181.

Batthyány, K., \& Cabrera, M. (2011). Metodología de la investigación en Ciencias Sociales. Udelar, CSE.

Becattini, E. (2018). When we were young: Netflix and the teen audience. Comunicazioni Sociali, 2018, 207-217.

Bellón Sánchez de la Blanca, T. (2012). Nuevos modelos narrativos. Ficción televisiva y transmediación. Revista Comunicación, 1(10), 17-31.

Berelson, B. (1952). Content analysis in communication research. Free Press.

Brantner, C., \& Rodriguez-Amat, J. R. (2016). New "danger zone" in europe: Representations of place in social media-supported protests. International Journal of Communication, 10(1), 299-320.

Cabezuelo-Lorenzo, F., Barrientos-Báez, A., \& Caldevilla-Domínguez, D. (2021). Propuesta para la transferencia del conocimiento e innovación en la enseñanzaaprendizaje del liderazgo: lecciones de cine. In Alfabetización en la nueva docencia. Editorial Tirant lo Blanch.

Cabezuelo-Lorenzo, Francisco, Barrientos-Báez, A., \& Caldevilla-Domínguez, D. (2020). Ficción audiovisual contemporánea como herramienta educativa en la actual sociedad multipantallas. Utopía y Praxis Latinoamericana. Revista Internacional de Filosofía y Teoría Social, 25(EXTRA 13), 210-224. https://doi.org/http://doi.org/10.5281/zenodo.4292728

Castillo-Pomeda, J. M. (2016). Connected. the Fourth Screen As Epicenter of Social Communications. Revista de Comunicación de La SEECI, 40, 1-17.

Fernández-Gómez, E., \& Martín-Quevedo, J. (2018a). Connecting with audiences in new markets: Netflix's Twitter strategy in Spain. Journal of Media Business Studies, 15(2), 127-146. https://doi.org/10.1080/16522354.2018.1481711

Fernández-Gómez, E., \& Martín-Quevedo, J. (2018b). La estrategia de engagement de Netflix España en Twitter. El Profesional de La Información, 27(6), 1292. https://doi.org/10.3145/epi.2018.nov.12

Gabriel, P. S., Carabaza González, J. I., \& Romo Gutiérrez, D. O. (2015). El uso de Twitter en las salas de redacción: redes sociales y newsmaking en la región sureste de Coahuila, México. Mediaciones Sociales, 0(12), 108-128. https://doi.org/10.5209/rev meso.2013.n12.45265 
Martínez Sala, A. M., Barrientos-Báez, A. y Caldevilla-Domínguez, D. Fandom televisivo.

Estudio de su impacto en la estrategia de comunicación en redes sociales de Netflix.

Gil-Quintana, J., \& Amoros, M. F. G. (2020). Posts, interactions, truths and lies of Spanish adolescents on Instagram. Texto Livre, 13(1), 20-44. https://doi.org/10.17851/1983-3652.13.1.20-44

González Neira, A., \& Quintas Froufe, N. (2016). El comportamiento de la audiencia lineal, social y en diferido de las series de ficción española. Revista de La Asociación Española de Investigación de La Comunicación, 3(6), 27-33. https://doi.org/10.24137/raeic.3.6.4

Harrington, S. (2014). Tweeting about the Telly: Live TV, Audiences, and Social Media. In K. Weller, A. Bruns, J. Burgess, M. Mahrt, \& C. Puschmann (Eds.), Twitter and Society (pp. 237-247). Peter Lang.

Harrington, S., Highfield, T., \& Bruns, A. (2013). More than a Backchannel: Twitter and Television. Participations: Journal of Audience \& Reception Studies, 10(1), 405-409.

Herrero Subías, M., \& Urgellés Molina, A. (2018). Generación de engagement con un servicio de vídeo bajo demanda en Twitter. El caso de Netflix España. Revista Comhumanitas, $9(2)$,

175-191. https://doi.org/https://doi.org/10.31207/rch.v9i2.172

Jenkins, H. (2010). Piratas de textos. Fans, cultura participativa y televisión. Paidós.

Jost, F. (2014). Webseries y series de tv: idas y venidas. Narraciones en tránsito. CIC Cuadernos de Información y Comunicación, 19(0), 39-51. https://doi.org/10.5209/rev ciyc.2014.v19.43902

Lacalle, C., \& Castro, D. (2018). Fandom televisivo y construcción de identidad. Análisis de los comentarios de las fans españolas y los community managers. Revista Latina de Comunicacion Social, 2018(73), 1-18. https://doi.org/10.4185/RLCS-2018-1242

Marcos, N. (2015). Una serie para la generación Twitter. In C. Cascajosa-Virino (Ed.), Dentro de El ministerio del tiempo (pp. 199-202). Léeme Libros.

Marta-Lazo, C., \& Gabelas-Barroso, J. A. (2013). Fiction Consumption Habits Among Communication. Revista de Comunicación de La SEECI, 31, 14-33.

Martínez-Sala, A.-M., \& Prado-Jiménez, C.-E. (2019). El papel del fandom televisivo en la estrategia de comunicación digital de Netflix. Análisis de la interacción y engagement online en Twitter. In V. Rodríguez Breijo, M. Trenta, S. Toledano Buendía, C. Hernández, A. Ardèvol Abreu, A. Álvarez Nobell, L. Morales, T. Hidalgo-Marí, \& A. Barrientos-Báez (Eds.), Las nuevas narrativas en el entorno social (pp. 111-112). Sociedad Latina de Comunicación Social. 
Martínez Sala, A. M., Barrientos-Báez, A. y Caldevilla-Domínguez, D. Fandom televisivo.

Estudio de su impacto en la estrategia de comunicación en redes sociales de Netflix.

Martínez-Sala, A.-M., \& Segarra-Saavedra, J. (2020). Engagement y disengagement online, factores clave en las estrategias de comunicación turística 2.0. In V. Altamirano-Benítez, M. Túñez-López, \& I. Marín-Gutiérrez (Eds.), Tendencias de la Comunicación para el Turismo (pp. 149-183). Editorial Dykinson.

Marwick, A. E., \& Boyd, D. (2011a). I tweet honestly, I tweet passionately: Twitter users, context collapse, and the imagined audience. New Media \& Society, 13(1), 114-133. https://doi.org/10.1177/1461444810365313

Marwick, A. E., \& Boyd, D. (2011b). To see and be seen: Celebrity Practice on Twitter. Convergence: The International Journal of Research into New Media Technologies, 17(2), 139-158. https://doi.org/10.1177/1354856510394539

Mayorga Escalada, S. (2019). Netflix, estrategia y gestión de marca en torno a la relevancia de los contenidos. AdComunica. Revista Científica de Estrategias, Tendencias e Innovación En Comunicación, 18, 219-244. https://doi.org/10.6035/2174-0992.2019.18.11

Miranda-Galbe, J., \& Cabezuelo-Lorenzo, F. (2018). La importancia de la documentación histórica en el relato transmedia: el caso de "El Ministerio del Tiempo." Revista de Ciencias de La Comunicación e Información, 23(1), 15-27. http://doi.org/10.35742/rcci.2018.23(1).15-27

Miranda Galbe, J., \& Figuero Espadas, J. (2016). El rol del prosumidor en la expansión narrativa transmedia de las historias de ficción en televisión: el caso de 'El Ministerio del Tiempo.' Index.Comunicación, 6(2), 115-134.

Monserrat-Gauchi, J., \& Martínez-Sala, A.-M. (2016). Cambio de paradigma en la interacción de las empresas con sus públicos. La generación de contenidos a través de Facebook. In M. Mut, A. Oliveira, R. Alberto Pérez, \& J. Xifra (Eds.), Casos de estudio de Relaciones Públicas. Sociedad conectada: empresas y universidades (pp. 59-85). Editorial UOC. Colección DIRCOM.

Monserrat-Gauchi, J., Quiles-Soler, M.-C., \& Martínez-Sala, A.-M. (2017). La participación ciudadana en la comunicación de las organizaciones. Análisis de las franquicias de salud-belleza, decoración y restauración. Prisma Social, 18, 540560.

Morimoto, S. A., \& Friedland, L. A. (2011). The lifeworld of youth in the information society. Youth and Society, 43(2), 549-567. https://doi.org/10.1177/0044118X10383655

Muruzábal, A., \& Grandío, M. (2009). La representación de la guerra en la ficción televisiva norteamericana contemporánea;Representation of War in Contemporary American Television Fiction. Mediaciones Sociales, 5, 63-83. https://doi.org/10.5209/rev MESO.2009.n5.22324 
Martínez Sala, A. M., Barrientos-Báez, A. y Caldevilla-Domínguez, D. Fandom televisivo.

Estudio de su impacto en la estrategia de comunicación en redes sociales de Netflix.

Navar-Gill, A. (2018). From Strategic Retweets to Group Hangs: Writers' Room Twitter Accounts and the Productive Ecosystem of TV Social Media Fans. Television and New Media, 19(5), 415-430. https://doi.org/10.1177/1527476417728376

Neira, E. (2015). Audiencia social:¿consiguen las redes sociales que veamos más televisión? In N. Quintas Froufe \& A. González Neira (Eds.), La participación de la audiencia en la televisión: de la audiencia activa a la social (pp. 47-59). AIMC, Asociación para la Investigación de Medios de Comunicación.

Neira, E. (2018). Impacto del modelo Netflix en el consumo cultural en pantallas: Big Data, susrcripción y long tail. Anuario AC/E de Cultura Digital 2018, 68-79.

Pavón-Arrizabalaga, A., Zuberogoitia, A., Astigarraga, I., Unibertsitatea, M., \& Juaristi, P. (2016). Consumo de series de televisión de los adolescentes en la era de la digitalización audiovisual: prácticas y motivaciones. Dígitos, Revista de Comunicación Digital, 2(2), 35-52.

Piñeiro-Otero, T. (2020). Flash Gordon. La expansión del héroe intergaláctico como ejemplo de arqueología transmedia. Historia y Comunicación Social, 25(1), 45-56. https://doi.org/10.5209/hics.69226

Prensky, M. (2001). Digital Natives, Digital Immigrants. On the Horizon, 9(5), 1-6. https://doi.org/10.1108/10748120110424816

Quintas-Froufe, N., \& González-Neira, A. (2014). Audiencias activas: participación de la audiencia social en la televisión. Comunicar, XXII(43), 83-90. https://doi.org/10.3916/C43-2014-08

Ramos Méndez, D., \& Ortega-Mohedano, F. (2017). La revolución en los hábitos de uso y consumo de vídeo en teléfonos inteligentes entre usuarios Millenials, la encrucijada revelada. Revista Latina de Comunicacion Social, 72, 704-718. https://doi.org/10.4185/RLCS-2017-1187

Rodríguez Pastene-Vicencio, F. (2017). Pasado presente. La construcción del tiempo en las narrativas transmediáticas. El caso de cuentosdeviejos.com. Perspectivas de La Comunicación, 10(2), 189-194.

Rubio Gil, Á. (2010). Generación digital: patrones de consumo de Internet, cultura juvenil y cambio social. Revista de Estudios de Juventud, 88, 201-221.

Santamaria López, T. M., Lagos Reinoso, G., Armijos Acosta, R., \& Bravo Villagómez, F. (2019). Streaming en vivo enfocado en el aprendizaje personalizado de la asignatura Programación mediante la red social Facebook. Revista Inclusiones, 6, 169-177. 
Martínez Sala, A. M., Barrientos-Báez, A. y Caldevilla-Domínguez, D. Fandom televisivo.

Estudio de su impacto en la estrategia de comunicación en redes sociales de Netflix.

Scolari, C. A. (2013). Narrativas transmedia. Cuando todos los medios cuentan. Deusto.

Segado-Boj, F., Grandío, M. D. M., \& Fernández-Gómez, E. (2015). Social media and television: A bibliographic review based on the web of science. Profesional de La Informacion, 24(3), 227-234. https://doi.org/10.3145/epi.2015.may.03

Torregrosa-Carmona, J.-F., \& Rodríguez-Gómez, E. (2017). Comunidades de fans y ficción televisiva. Estudio de caso: El ministerio del tiempo (TVE). El Profesional de La Información, 26(6), 1139. https://doi.org/10.3145/epi.2017.nov.13

Ugalde Lujambio, L., Martínez, J., \& Medrano Samaniego, M. (2017). Pautas de consumo televisivo en adolescentes de la era digital: un estudio transcultural. Comunicar: Revista Científica Iberoamericana de Comunicación y Educación, 50, 67-76.

\section{AUTORES:}

\section{Alba-María Martínez-Sala}

Dra. en Bellas Artes (Arte y diseño gráfico) y Premio Extraordinario de Doctorado de la Universidad Miguel Hernández (2015-2016). Licenciada en Ciencias de la Información, Publicidad y Relaciones Públicas (Universidad Complutense de Madrid). Es profesora Ayudante Doctora (acreditada a Contratada Doctora por la ANECA y AVAP) en la Universidad de Alicante (UA), en el departamento de Comunicación y Psicología Social (Grado oficial y Másteres oficiales). Sus principales líneas de investigación se centran en el área de las estrategias de comunicación y marketing digital tanto en el ámbito público como en el privado. Pertenece a los grupos de investigación MAE-CO y AgeCOM (UA) y GCE (Universidad Técnica Particular de Loja, Ecuador). Ha sido distinguida como profesora honorífica por la Universidad Católica de Miami (EE. UU.)

albamaria.martinez@ua.es

Orcid ID: https://orcid.org/0000-0002-6852-6258

Google Scholar: https://scholar.google.es/citations?hl=es\&user=09- 0goAAAA]

ResearchGate: https://www.researchgate.net/profile/Alba Maria Martinez-Sala

Scopus: 57194398505

\section{Academia.edu:}

https://independent.academia.edu/AlbaMar\%C3\%ADaMart\%C3\%ADnezSala

\section{Almudena Barrientos-Báez}

Dra. con mención internacional en Educación (profesora acreditada contratada doctor). Profesora en la Escuela Universitaria de Turismo Iriarte, adscrita a la ULL y en la Univ. Europea. Máster en Dirección de Protocolo, Producción, Organización y Diseño de Eventos - área de Comunicación - (UCJC) y Máster en Dirección de Alojamientos Turísticos (Univ. Girona). Grado en Turismo (EUTI-ULL) y Magisterio (Univ. Valencia). 
Forma parte del proyecto DEBATV, Debates Electorales Televisados en España: Modelos, Proceso, Diagnóstico y Propuesta (CSO2017-83159-R), proyecto de I+D+I, y del proyecto Nuevos valores, gobernanza, financiación y servicios audiovisuales públicos para la sociedad de Internet: contrastes europeos y españoles (RTI2018096065-B-I00) de investigación del Programa Estatal de I+D+I orientado a los Retos de la Sociedad del Ministerio de Ciencia, Innovación y Universidades (MCIU), Agencia Estatal de Investigación (AEI) y del Fondo Europeo de Desarrollo Regional (FEDER). almudenabarrientos@iriarteuniversidad.es

Orcid ID: https://orcid.org/0000-0001-9913-3353

Google Scholar: https://scholar.google.es/citations?user=jUHzsfIAAAAJ\&hl=es

\section{David Caldevilla-Domínguez}

Licenciado y Doctor en Ciencias de la Información (Comunicación Audiovisual por la U. Complutense). Diplomado en Magisterio (U. de Zaragoza). Profesor titular en la Facultad de Ciencias de la Información de la UCM. Docente en: U. Complutense, U. Europea de Madrid, IED, ESERP e IPAM (Oporto -Portugal-). Índice h $16.6^{\circ}$ autor español en trabajos publicados, $13^{\circ}$ en artículos citados y $20^{\circ}$ en citas recibidas de 747 autores totales.

davidcaldevilla@ccinf.ucm.es

Orcid ID: https://orcid.org/0000-0002-9850-1350

Google Scholar: https://scholar.google.es/citations?user=wVd-AnwAAAAJ\&hl=es 